Journal of Computer Science (Special Issue): 89-91, 2005

ISSN 1549-3636

(C) 2005 Science Publications

\title{
Neurocomputing Device for Image Processing and Classification
}

\author{
Rashad J. Rasras
}

Faculty of Engineering Technology, P.O.Box 15008, Amman- Jordan

\begin{abstract}
This article is concerned with problems of construction of specialized computing device for image processing and classification. The device may be used for classification of objects images in conditions of various image scales of the same object. The device may be realized by neurochips technology.
\end{abstract}

Key words: Neurocomputing device, image processing, classification

\section{INTRODUCTION}

The problem of classification of the object images $\mathrm{A}_{\mathrm{k}}(x, y), k=\overline{1, m_{0}}$ at their free position in the area of vision $D(x, y)$ and various scale images $m_{j r}, j=\overline{1, m}, r=\overline{1, R_{j}}$; where $\mathrm{m}$ - is the number of different objects presented in different scales; $R_{j}$-is the number of different scales of $\mathrm{j}$ - object images.

Assume that we have in the vision area $D(x, y)$ images $S(x, y)$, which have the background $A_{\Phi}(x, y)$ and $\mathrm{m}_{0}$ of objects; where $m_{0}=\sum_{j=1}^{m} \sum_{r=1}^{R_{j}} j_{r}$. Let $D_{\phi} \subset D$ be an area of background; and $D_{k} \subset D, k=\overline{1, m_{0}}$ be the area of the $\mathrm{k}_{\mathrm{th}}$ object, knowing that objects images don't intersect.

$D_{i} \cap D_{n}=\phi$ where $i \neq n$

And satisfy the condition:

$D_{\phi} \cup D_{1} \cup D_{2} \cup \ldots \cup D_{m_{0}}=D$.

A set of background images $S_{\Phi}(x, y)$ and separate different objects $S_{k}(x, y), k=\overline{1, m_{0}}$ forms the image:

$S(x, y)=S_{\Phi}(x, y) \cup S_{1}(x, y) \cup \ldots \cup \operatorname{Sn}(x, y)$,

$S_{i} \cap S_{n}=\phi$, if $i \neq n$.

Satisfying the conditions 1-3 means that:

$S_{\phi}(x, y)=D_{\Phi}(x, y)$ where $(\mathrm{x}, \mathrm{y}) \in D_{\Phi}$,

$S_{k}(x, y)=D_{k}(x, y)$ Where $(\mathrm{x}, \mathrm{y}) \in D_{k}, k=\overline{1, m_{0}}$.

On solving the problem of classification performed on the image $S(x, y)$ of multi $m_{0}$ objects, into unknown in advance number of various classes. First stage of processing is procedure of segmentation of the image $\mathrm{S}(\mathrm{x}, \mathrm{y})$, mathematically segmentation procedure may be described by the evaluation of the following predicate:

$P(x, y)=\left\{\begin{array}{l}k, \text { if }(x, y) \in D_{k}, \\ 0, \text { if }(x, y) \in D_{\phi} .\end{array}\right.$

The result of predicate (4), the source image in equation (3) is segmented into $\mathrm{m}_{0}$ number of different separate images $S_{k}(x, y), k=\overline{1, m_{0}}$, the part of which differs from each other only by scale (they are images of the same object).
The main part: To determine the class of each image in the vision area in situation of different scale of objects images, it is rational to use space feature indicator which contains the following parameters perimeter $\mathbf{P}_{\mathbf{k}}$ and area $S_{k}^{\text {area }}$ of images objects, it is known in work ${ }^{[1]}$ that the ratio:

$$
\rho=\frac{P}{\sqrt{S^{\text {area }}}}
$$

Notice that $P$ and $S_{k}^{\text {area }}$ are invariant to the changes of the scale of any polygon. As in the work ${ }^{[1]}$ the equation (5) is given with out proofs, so let us show its correctness for any shape. For squares with length a and wide $b$, applying equation of the ratio (5) we get:

$\rho_{0}=\frac{2(a+b)}{\sqrt{a b}}$.

On changing the image scale in $\mathrm{k}$ times $(0<k<\infty)$, $a_{1}=k a ; b_{1}=k b$; we get the following:

$\rho_{1}=\frac{2\left(a_{1}+b_{1}\right)}{\sqrt{a_{1} b_{1}}}=\frac{2(k a+k b)}{\sqrt{k^{2} a b}}=\rho_{0}$.

We see that the ratio (5) is invariant to the change of square scale. Now let us show that the invariant of the ration (5) to the scale changes for any shape. In Fig. 1 is shown that any figure may be shown as a sum of squares the area of which aims to zero.

For shape $\mathrm{A}(\mathrm{x}, \mathrm{y})$, the ratio (5) may be given in the following equation:

$\rho_{A}=\frac{P_{A}}{\sqrt{S_{A}{ }^{\text {area }}}}=\frac{a_{1}+a_{q}+2 \sum_{i=1}^{q} b_{i}}{\sqrt{\sum_{i=1}^{q} a_{i} b_{i}}}$,

where $a_{1}, a_{q}$ - are the length of the first and last squares; $b_{i}(i=\overline{1, q})$ - are the width of squares.

On changing the scale of the image $\mathrm{A}(\mathrm{x}, \mathrm{y})$ in $\mathrm{k}$ times, we have

$\rho_{k}=\frac{P_{k}}{\sqrt{S_{k}^{\text {area }}}}=\frac{k a_{1}+k a_{q}+2 \sum_{i=1}^{q} k b_{i}}{\sqrt{\sum_{i=1}^{q} k^{2} a_{i} b_{i}}}=\frac{a_{1}+a_{q}+2 \sum_{i=1}^{q} b_{i}}{\sqrt{\sum_{i=1}^{q} a_{i} b_{i}}}=\rho$,

Corresponding Author: Rashad J. Rasras, Faculty of Engineering Technology, P.O.Box 15008, Amman- Jordan 
where $\mathrm{P}_{\mathrm{k}}$ and $S_{k}^{\text {area }}$-are correspondingly perimeter and area of the figure, the scale of which is changed in $\mathrm{k}$ times.

Thus, the ratio (5) is invariant for any shape presented in different scale.

To calculate the area of k-object image, which is in the area of vision $\mathrm{D}(\mathrm{x}, \mathrm{y})$ using predicate (4) it is enough to calculate the number $\mathrm{N}_{\mathrm{k}}$ of pixels forming the image and multiply it by the area $S_{p}$ of one pixel:

$S_{k}^{\text {area }}=N_{k} \cdot S_{p}$.

To calculate the perimeter of any $\mathrm{k}$ image, we consider that it consists of the length of separate pixels. As shown in Fig. 2 any pixel of the image may have one of the following numbers of adjacent pixels.

Pixels marked on Fig. 2 by the digit 1 , have 4 neighboring pixels (up, down, right, left), the contribution of theses pixels to the length of perimeter is equal zero.

Pixels marked on Fig. 2 by the digit 2, each have three neighboring pixels; their contribution to the perimeter length is equal to the length of one pixel $1 \mathrm{~d}$.

Pixels marked on Fig. 2 by the digit 3, they have two neighboring pixels; their contribution to the perimeter length is equal to $2 \mathrm{~d}$.

Pixels marked on Fig. 2 by the digit 4 have only one neighboring pixel; their contribution to the perimeter length is equal to $3 \mathrm{~d}$.

Pixels marked on Fig. 2 by the digit 5; their contribution to the perimeter length is equal to $4 \mathrm{~d}$.

By analyzing Fig. 2 and the given reasoning, we can calculate the length of perimeter of any image by the following:

$P=N_{1} d+2 N_{2} d+3 N_{3} d+4 N_{0} d$,

Where $N_{r}, r=\overline{1,3}$ is the number of excited neurons which have $\mathrm{r}$ neighboring excited pixels in the image; $\mathbf{N}_{\mathbf{0}}$ is the number of neurons which don't have neighboring pixels in their space.

Images classification using ratio (5), it is rational to be carried out with the help of technology of neural networks. One of the possible architecture is given in Fig. 3. The device has the area of vision $D(x, y)$ from matrix $\mathrm{n}_{1} \times \mathrm{n}_{2}$ of binary $\mathrm{x}$ neurons, where $\mathrm{n}_{1}-$ number of rows, $\mathrm{n}_{2^{-}}$number of columns. Every $\mathrm{x}$-neuron corresponds to one pixel of the image $\mathrm{D}(\mathrm{x}, \mathrm{y})$.

The function of X-neurons may be described by the following formula:

$U_{\text {out }}=\left\{\begin{array}{l}1, \text { if } \quad \text { Uin } \geq \theta_{n}, \\ 0, \text { if } U_{\text {in }} \leq \theta_{n},\end{array}\right.$

Where $\mathrm{U}_{\text {in }} ; \mathrm{U}_{\text {out }}-$ are correspondingly input and output signals of X-neurons; $\theta_{n}$ - Threshold value of Xneurons.

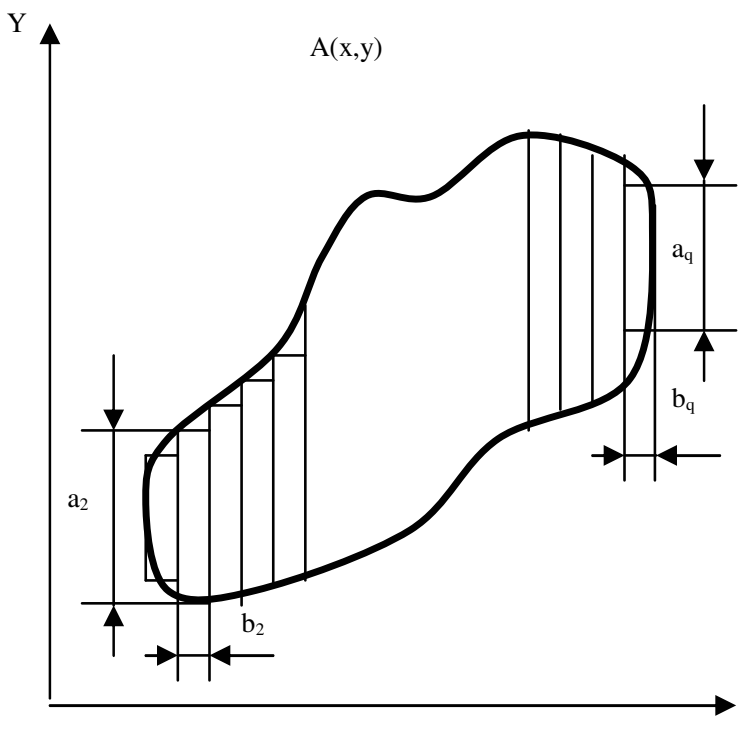

Fig. 1: Shape A as a sum of squares the area of which aims to zero.

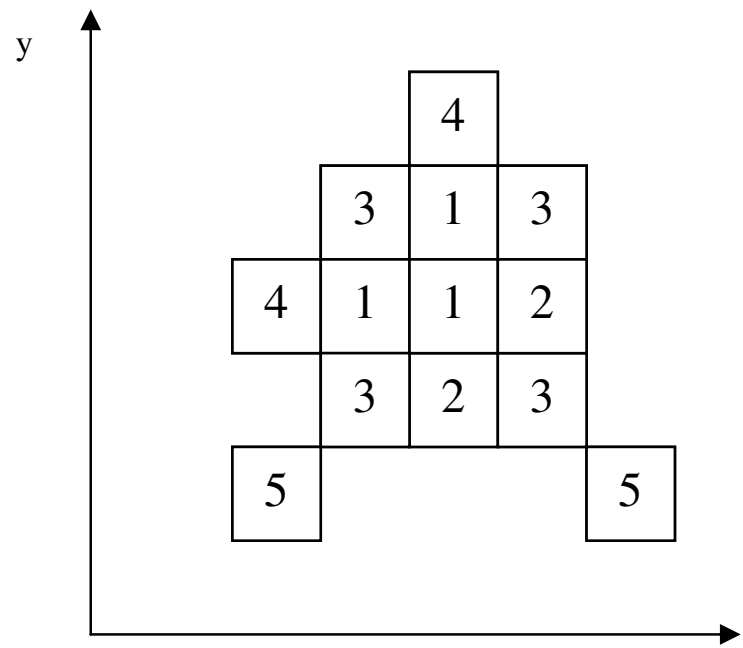

Fig. 2: Contribution to of different Pixels ${ }^{\mathrm{X}}$ to the perimeter length of image.

Neurons in layer fields $z^{1}-z^{4}$ receive binary signals from output of $\mathrm{x}$-neurons layer, each neuron of $Z_{i j}^{k}\left(k=\overline{1,4}, i=\overline{1, n_{1}}, j=\overline{1, n_{2}}\right)$ receives input signals from $\mathrm{x}_{\mathrm{ij}} \quad$ and $\quad$ its neighbors; $x_{i-1, j}(i-1 \geq 1), x_{i+1, j}\left(i+1 \leq n_{1}\right), x_{i, j-1}(j-1 \geq 1), x_{i, j+1}\left(j+1 \leq n_{2}\right)$

The value of output signals from neurons of field $\mathrm{Z}^{\mathrm{k}}$ may be determined by the following formula:

$U_{\text {out } Z_{i j}^{k}}=\left\{\begin{array}{l}1, \text { if } U_{\text {in } Z_{i j}^{k} \geq \theta^{k}}, \\ 0, \text { if } U_{\text {in } Z_{i j}^{k} \theta^{k}},\end{array}\right.$

Where $U_{i n Z_{i j}^{k}}$ - input signal of neurons $Z_{i j}^{k}, i=\overline{1, n_{1}}, j=\overline{1, n_{2}}$. 


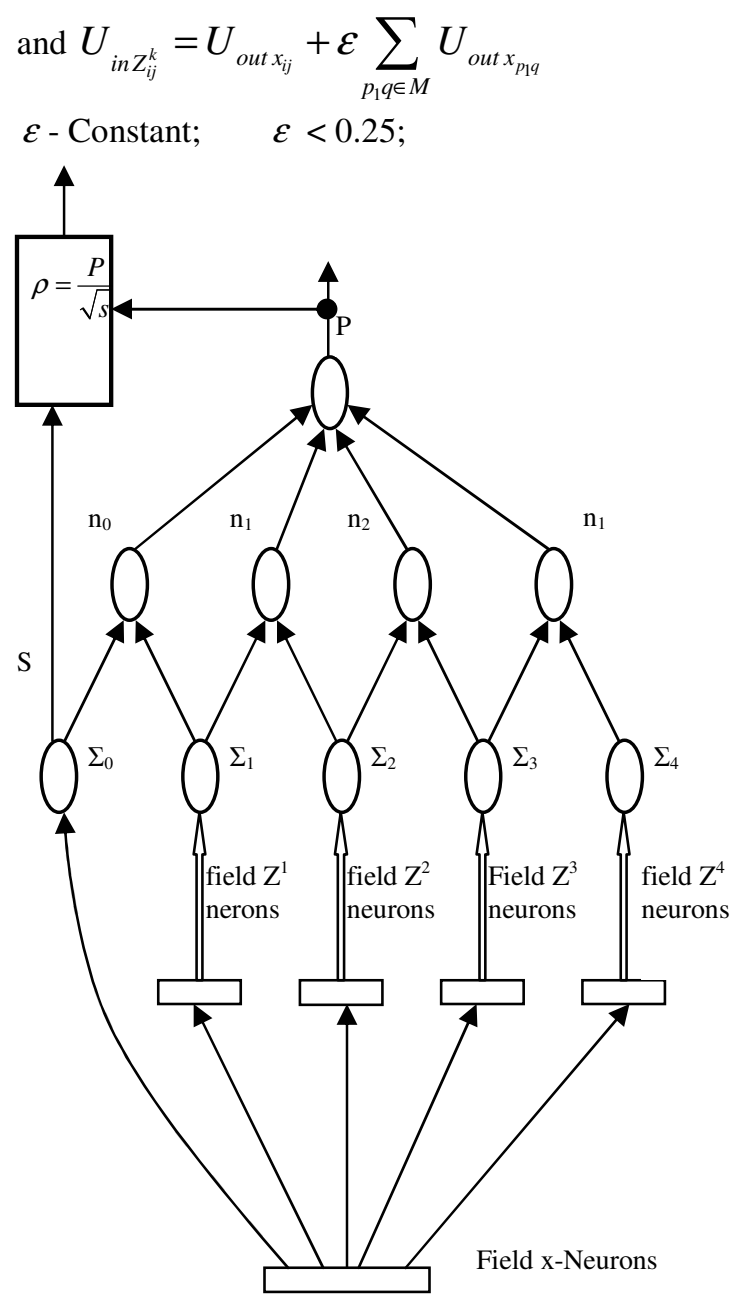

Fig. 3: Neurocomputing Device Architecture

M- a set of indexes determining neighboring neurons of $\mathrm{x}_{\mathrm{ij}}$ neuron.

$\theta^{k}$ - The threshold value of neurons in fields $\mathrm{Z}^{\mathrm{k}}, \theta^{k}=1$ $+\varepsilon k, \mathrm{k}=1,4$.

Analysis of equation (8) shows that binary neurons of field $Z_{i j}^{1}$ are excited (fired) if neuron $\mathrm{x}_{\mathrm{ij}}$ is excited and it has from one to four neighboring neurons which have output signal of one value. Analogously, neurons $Z_{i j}^{k}$ of the field $Z^{k}(k=2,3,4)$ get into the excited condition if the neuron $\mathrm{x}_{\mathrm{ij}}$ is excited and it has $\mathrm{k}$ neighboring neurons which have output signal of one value. neurons $\sum_{p}, p=\overline{0,4}$ have linear activation function; the output signal value may be determined by the following formula:

$U_{\text {out } \sum_{p}}=k_{1} \sum_{i=1}^{n_{1}} \sum_{j=1}^{n_{2}} U_{\text {out } Z_{i j}^{p}}, p=\overline{0,4}$,

where $\mathrm{k}_{1}$ - is a positive constant; $U_{\text {out } Z_{j}^{p}}$ - is output signal of the neurons $Z_{i j}^{p}$ of p-field.
Thus, the output signal will be signal proportional to the general number of the excited $\mathrm{x}$-neurons and the area of given image. When output signal of a neuron $\sum_{q}(q=\overline{1,4})$ is proportional to the number of elements of the image which have from $\mathrm{q}$ to four excited conditions. Using the equation (9), it is easy to receive the signals proportional to number $\mathrm{N}_{0}$ of neurons which don't have neighboring elements on the image and signals proportional to numbers $\mathrm{N}_{\mathrm{r}}(r=\overline{1,3})$ of neurons which has correspondingly from one to three excited elements in the image

$$
\begin{aligned}
& N_{0}=k_{1}\left(U_{\text {out } x} \sum_{0}-U_{\text {out } \sum_{1}}\right), \\
& N_{1}=k_{1}\left(U_{\text {out } \sum_{1}}-U_{\text {out } \sum_{2}}\right), \\
& N_{2}=k_{1}\left(U_{\text {out } \sum_{2}}-U_{\text {out } \sum_{3}}\right), \\
& N_{3}=k_{1}\left(U_{\text {out } \sum_{3}}-U_{\text {out } \sum_{4}}\right) .
\end{aligned}
$$

the right parts of the ratio (10) in computing device are carried out with the help of neurons $n_{q}(q=\overline{0,3})$, which have linear activation function.

Having signals proportional to the area and perimeter of the image, it is easy to receive signal as well with the help of ALU block, which will be proportional to the first part of equation (5).the resultant value of signal may be used to classify input image.

\section{RESULTS}

Experiments conducted on image like given in Fig. 4 prove the ability to work with the offered algorithm. The developed device is suggested to apply for speeding up image process in different fields of applications.

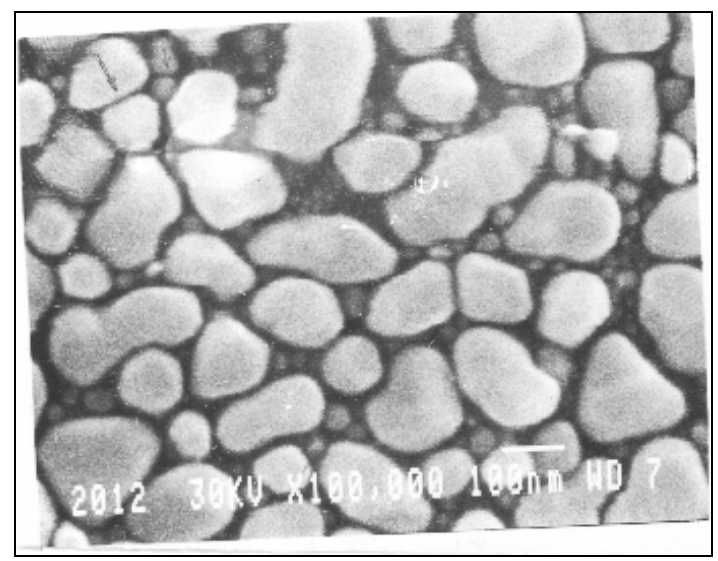

Fig. 4: Processed Image

\section{REFERENCES}

1. Feder E. Fractals. M. Mir, 1991. pp: 254.

2. kroglov, V., 2001. Artificial Neural Networks. Theory and Practice. M. Gorachaya Linia-Telecom, pp: 382. 